\section{Patrimonio, turismo y desarrollo sustentable: \\ caso costa de Jalisco}

DOI: 10.22403/UQROOMX/TYP02/01

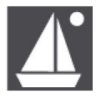

Rosa María Chávez Dagostino

Edmundo Andrade Romo

Rodrigo Espinoza Sánchez

\title{
RESUMEN
}

La costa de Jalisco constituye un factor determinante que incide en el desarrollo regional, no obstante, presenta un estado de desintegración. Está comprendida por cinco municipios que conforman el corredor turístico llamado Costa Alegre, donde coexisten niveles desde extrema pobreza hasta desarrollados.

Se planteó la necesidad de realizar un inventario de recursos naturales, culturales y productivos en la región costa de Jalisco, con la finalidad de incluirlos en un uso turístico diferente al modelo que presiona bipolarmente esta región, y promover a la vez la integración de este corredor a través de valores distintos: naturaleza, cultura y producción.

Se hace énfasis en la importancia y la metodología empleada. Asimismo, se discute la integración de la actividad turística a otras actividades económicas, como eje vertebrador para el desarrollo local, que coadyuve en el bienestar social y calidad de vida.

Palabras Atractivo turístico, calidad de vida, desarrollo sustentable, inventario, CLAVE recursos, región.

* Universidad de Guadalajara / rosac@pv.udg.mx I edmundoa@pv.udg.mx / rodrigoe@pv.udg.mx 


\section{Introducción}

El estudio de regiones asociadas al turismo implica un análisis de desarrollo particular, con características comunes y diferencias importantes entre las distintas regiones relacionadas con la actividad turística.

Uno de los factores más significativos que confieren particularidad se basa en los recursos regionales, sean naturales, culturales o productivos, por lo que su estudio e inventario resulta de suma importancia.

El conjunto de bienes o recursos regionales, a la postre conforman el patrimonio regional; su empleo, uso o incorporación en la actividad turística nos permite, en un primer plano, predeterminar una región turística por sus atractivos; sin embargo, existen otros factores que inciden propiamente en el concepto de desarrollo regional, máxime si se presume que es sustentable.

En México, las regiones turísticas (desarrolladas o en desarrollo) distan mucho de ser o aproximarse a lo sustentable, que en principio está estrictamente vinculado con el estado de bienestar de las poblaciones locales y con la no depredación del medio natural.

La región de estudio se circunscribe a la costa de Jalisco, formada por cinco municipios que son, de norte a sur, Puerto Vallarta, Cabo Corrientes, Tomatlán, La Huerta y Cihuatlán.

Desde la perspectiva del desarrollo, la sustentabilidad y el turismo, que ya de por sí son una trípode de moda bastante compleja, el concepto de región debe partir, al menos para su análisis, de la noción de dinamismo y de la consideración de que es una resultante multifactorial.

\section{Antecedentes}

Actualmente, los estudios de regionalización en Jalisco han subdividido su territorio en 12 regiones, para efectos de atención pública y apoyos nacionales o estatales de programas de desarrollo económico (Gobierno del Estado de Jalisco, 2000a y 2000b); en tanto, lo político a nivel estatal está distribuido en 20 distritos. Nuestra zona de estudio comprende dos regiones y dos distritos (región 9 y $5^{\circ}$ distrito; región 8 y $18^{\circ}$ distrito).

Además, la región costa de Jalisco se encuentra enclavada en tres subregiones, una de carácter histórico-cultural que la vincula más hacia el sur con los estados de Colima y Michoacán; otra, de índole geográfica y natu- 
ral, denominada región de Bahía de Banderas en el norte, y vincula la parte norte de la costa de Jalisco con el litoral costero sur del estado de Nayarit; $y$ una tercera, de condición mixta, y que une la costa de Jalisco con los municipios serranos Talpa de Allende, San Sebastián del Oste, Mascota, Casimiro Castillo, Cuautitlán, Cuautla, y Villa de Purificación, a partir de las cuencas y de los beneficios mineros del siglo XVIII Y XIX.

Los estudios realizados por el Instituto Nacional de Estadística, Geografía e Informática (INEGI) permiten caracterizar la región con base en su demografía, medio ambiente, servicios públicos e ingresos (cuadro I), estos datos nos dan un parámetro de las disimilitudes. Se infiere que la región costa de Jalisco está polarizada, esto es, que el desarrollo se expresa de manera muy significativa en sus extremos norte y sur: Puerto Vallarta y Barra de Navidad-Melaque. Esta dinámica incide más en las localidades de Nuevo Vallarta y Colimilla (en Nayarit y Colima, respectivamente) ya que su crecimiento económico ha experimentado un auge a partir de dicha influencia, lo interesante aquí es que los municipios vecinos de otros estados son los beneficiados. El desarrollo turístico de los municipios costeros intermedios de Jalisco (Cabo Corrientes, Tomatlán y la Huerta) no han logrado aprovechar esta situación.

La región costa de Jalisco ha sido objeto de estudio de manera fragmentada. El Colegio de Jalisco publicó los trabajos del cronista de la ciudad de Puerto Vallarta, Carlos Munguía Fragoso, quien aporta información histórica, pero para el resto de los municipios los estudios son escasos, no todos cuentan con monografías o información oficial.

A partir de 1994, la Universidad de Guadalajara emprendió estudios regionales como el del Proyecto caracterización de la Bahía de Banderas (UDG, 1999); el trabajo sobre el municipio de Cabo Corrientes (Andrade-Romo, 1999), la compilación de artículos y estudios relacionados con la Bahía de Banderas en la revista México (2002), así como la descripción de playas de la bahía (López et al., 2002: 5-12). Con la participación del Rural Development Institute, Brandon University y el Centro Universitario de la Costa se realizaron estudios y una publicación, sobre impactos turísticos y calidad de vida de la región (Everitt, 200 I: I86-204; Massam, 2003) y, recientemente, un estudio sobre vocacionalidad turística de la costa de Jalisco, que no incluye a Puerto Vallarta (UDG, 2004).

El objetivo de realizar el inventario de recursos naturales, culturales y productivos en los municipios que integran la región costa norte de Jalisco: Puerto Vallarta, Cabo Corrientes, Tomatlán, La Huerta y Cihuatlán se justifica 
por tratarse de una zona con presión del desarrollo turístico, compleja, y sin el conocimiento de los recursos disponibles, lo que impide una buena planeación del desarrollo turístico y que las comunidades locales mejoren su calidad de vida mediante el uso de esos recursos.

\section{Metodología}

A fin de entender lo que incide en la problemática de la región costera o Costa Alegre de Jalisco se ideó un método que nos permitiera obtener la información suficiente, y luego sistematizarla para llegar a las primeras conclusiones.

Aspectos considerados para el análisis regional relacionado con turismo a nivel regional:

Simbología

I. Caracterización (Cr)

2. Conceptualización (Cn)

3. Estructura social (Es)

4. Problemática $(\mathrm{Pr})$

5. Estudio por municipio (Em)

a. Desarrollo turístico municipal

b. Estado de integración al corredor turístico

c. Análisis Fodas

d. Subregionalización

i. Costa

ii. Valle

iii. Sierra

6. Recursos
a. Naturales
b. Culturales
c. Productivos

7. Valoración o ponderación

8. Atractivos turísticos

9. Tipología turística

Es importante destacar que los estudios particulares realizados por municipios $(\mathrm{Em})$ permitieron generar supuestos para la formulación de una tendencia de la tipología turística $(\mathrm{Tt})$ y con ello unificar recursos, estructura 
municipal y actividad turística específica, que compita regionalmente, a la vez que complemente y fortalezca la región.

La actividad turística, en cualquiera de sus modalidades, no pretende competir con las otras actividades económicas en cada municipio, sino que se parte del supuesto de la incorporación para fortalecer el desarrollo local, mejorar el bienestar social y, por ende, elevar la calidad de vida.

La elaboración de la matriz donde se aplicaron los resultados del trabajo de campo en relación con la identificación de los recursos (naturales, culturales y productivos), así como la clasificación de variables y ponderación de las subvariables, está basada en los modelos de Sectur (s/f) y Martínez Tarragó (2000), a los que se les hicieron algunas modificaciones para los tres tipos de recursos.

Los trabajos de investigación, tanto documental como de campo, se realizaron a partir de 1998; el análisis de la información generada, los ajustes, la elaboración de primeros resultados así como productos, se llevó a cabo a principios del 2004.

\section{Resultados}

Con el fin de comprender mejor la incidencia de los recursos de un municipio o región se realizó un concentrado de información relativa a los aspectos más significativos de índole social, natural y económica.

En los siguientes cuadros ( 1,2 y 3 ) se expresan parte de los resultados de la investigación documental que nos permiten representar de manera esquemática la complejidad de la región e identificar los elementos más importantes a fin de cruzar la información; en una segunda etapa, se tomaron en cuenta tendencias, programas y proyectos, ya fueran públicos o privados.

De acuerdo con el cuadro 2, la región donde se enclava el área de estudio manifiesta una tendencia centro-sur; es homogénea en su estructura social con cierto equilibrio poblacional, territorial y tipológico; turísticamente se complementa y diversifica; sin embargo, es heterogénea en cuanto a su caracterización, conceptualización y problemática, en tanto que en el segmento centro-norte los municipios son totalmente heterogéneos.

La información sobre la actividad turística regional, según datos oficiales, muestra cómo dicha actividad zonifica la región, lo que resulta interesante ya que en primera instancia se puede observar una tendencia tipológica turística 
Patrimonio, turismo y

desarrollo sustentable: caso

costa de Jalisco

Cuadro I. Datos básicos de la región Costa Norte de Jalisco (1997)

\begin{tabular}{|c|c|c|c|c|c|c|}
\hline & $\begin{array}{l}\text { Puerto } \\
\text { Vallarta }\end{array}$ & $\begin{array}{c}\text { Cabo } \\
\text { Corrientes }\end{array}$ & Tomatlán & La Huerta & Cihuatlán & Región \\
\hline \multicolumn{7}{|l|}{ Demografía } \\
\hline Población & $18374 \mid$ & 9161 & 34220 & 22811 & 31850 & 281783 \\
\hline$\%$ estatal & 2.91 & 0.14 & 0.54 & 0.36 & 0.50 & 4.41 \\
\hline Superficie (ha) & 69397 & 153457 & 391594 & |74 97| & 74350 & 863739 \\
\hline$\%$ estatal & 0.87 & 1.94 & 4.95 & 2.21 & 0.94 & 10.94 \\
\hline $\begin{array}{l}\text { Comunidades } \\
\text { indígenas }\end{array}$ & - & 52 & 2 & 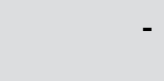 & - & 9 \\
\hline $\begin{array}{l}\text { Localidades mayores } \\
\text { de } 2500 \text { hab. }\end{array}$ & 4 & 12 & I & - & 3 & II \\
\hline $\begin{array}{l}\text { Localidades menores } \\
\text { de } 2500 \text { hab. }\end{array}$ & 92 & 132 & 196 & 134 & 58 & 612 \\
\hline \multicolumn{7}{|l|}{ Medio Ambiente } \\
\hline ANP* & I & I & - & 5 & - & 7 \\
\hline
\end{tabular}

SeRVICIOS PÚBLICOS

\begin{tabular}{|c|c|c|c|c|c|c|}
\hline Sin agua entubada (\%) & 5.0 & 46.0 & 27.3 & 14.6 & 10.8 & 20.74 \\
\hline Sin energía eléctrica (\%) & 2.84 & 47.3 & 20.4 & 8.36 & 5.6 & 16.9 \\
\hline $\begin{array}{l}\text { Sin drenaje a la } \\
\text { red pública (\%) }\end{array}$ & 19.6 & 89.2 & 71.9 & 81.3 & 45.9 & 61.5 \\
\hline \multicolumn{7}{|l|}{ INGRESOS } \\
\hline $\begin{array}{l}\text { Impuestos en } \\
\text { miles de pesos** }\end{array}$ & $22 ’ 594$ & 609 & 609 & 609 & 609 & $25^{\prime} 030$ \\
\hline $\begin{array}{l}\text { Participación federal } \\
\text { en miles de pesos** }\end{array}$ & $58^{\prime} 527$ & $3^{\prime} 182$ & $3^{\prime} 182$ & $3^{\prime} 182$ & $3^{\prime} 182$ & $7 I^{\prime} 263$ \\
\hline $\begin{array}{l}\text { Otros en miles } \\
\text { de pesos** }\end{array}$ & 13.404 & 712 & 712 & 712 & 712 & $16^{\prime} 252$ \\
\hline $\begin{array}{l}\text { Condición del } \\
\text { municipio }\end{array}$ & Desarrollado & $\begin{array}{l}\text { Extrema } \\
\text { pobreza }\end{array}$ & Pobreza & Pobreza & $\begin{array}{c}\text { En } \\
\text { desarrollo }\end{array}$ & Variada \\
\hline
\end{tabular}

Fuente: INEGI (1998). Anuario estadístico del estado de Jalisco, México: Gobierno del Estado de Jalisco.

* Se considera a la Costa de Alegre un corredor turístico ecológico.

**Las cifras de los municipios, excepto Puerto Vallarta, se calcularon a partir del total de rubro "el resto de municipios" entre 105 municipios no desglosados, resulta una cantidad equitativa como un supuesto por municipio. 
Cuadro 2. Concentrado de Resultados diagnóstico Regional

\begin{tabular}{|c|c|c|c|c|c|}
\hline Localidad & $\mathrm{Cr}$ & $\mathrm{Cn}$ & Es & $\mathrm{Pr}$ & $\mathrm{Em}(T \mathrm{t})$ \\
\hline $\begin{array}{l}\text { Puerto } \\
\text { Vallarta }\end{array}$ & $\begin{array}{l}\text { Destino turístico } \\
\text { internacional }\end{array}$ & $\begin{array}{l}\text { Contexto } \\
\text { urbano/ } \\
\text { desarrollado }\end{array}$ & $\mathrm{PP} / \mathrm{E}$ & $\begin{array}{l}\text { Falta de } \\
\text { espacios } \\
\text { urbanos }\end{array}$ & $\begin{array}{l}\text { Turismo masivo } \\
\text { Turismo cultural } \\
\text { Turismo de } \\
\text { negocios }\end{array}$ \\
\hline $\begin{array}{l}\text { Cabo } \\
\text { Corrientes }\end{array}$ & $\begin{array}{l}\text { Desintegrado/ } \\
\text { aislado }\end{array}$ & $\begin{array}{l}\text { Contexto } \\
\text { rural / } \\
\text { Extrema } \\
\text { pobreza }\end{array}$ & $\mathrm{PP} / \mathrm{E} / \mathrm{Cl}$ & $\begin{array}{l}\text { Tenencia } \\
\text { de la tierra / } \\
\text { Infraestructura }\end{array}$ & $\begin{array}{l}\text { Etnoturismo / } \\
\text { Ecoturismo }\end{array}$ \\
\hline Tomatlán & $\begin{array}{l}\text { Población } \\
\text { migrante }\end{array}$ & $\begin{array}{l}\text { Contexto } \\
\text { rural/ } \\
\text { Pobreza }\end{array}$ & $\mathrm{PP} / \mathrm{E} / \mathrm{Cl}$ & $\begin{array}{l}\text { Tenencia } \\
\text { de la tierra/ } \\
\text { siembra ilegal }\end{array}$ & $\begin{array}{l}\text { Turismo rural/ } \\
\text { Turismo depor- } \\
\text { tivo }\end{array}$ \\
\hline La Huerta & Fragmentación & $\begin{array}{l}\text { Contexto } \\
\text { rural/ } \\
\text { Pobreza }\end{array}$ & $\mathrm{PP} / \mathrm{E} / \mathrm{Cl}$ & $\begin{array}{l}\text { Espacio } \\
\text { geográfico }\end{array}$ & $\begin{array}{l}\text { Turismo de } \\
\text { aventura/ } \\
\text { Ecoturismo }\end{array}$ \\
\hline Cihuatlán & $\begin{array}{l}\text { Inversión } \\
\text { monopólica }\end{array}$ & $\begin{array}{l}\text { Contexto } \\
\text { semiurbano/ } \\
\text { en desarrollo }\end{array}$ & $\mathrm{PP} / \mathrm{E}$ & Promoción & $\begin{array}{l}\text { Turismo masivo } \\
\text { Turismo rural }\end{array}$ \\
\hline $\begin{array}{l}\text { Región } \\
\text { costa Jal. }\end{array}$ & Variada & Variada & $\mathrm{PP} / \mathrm{E} / \mathrm{Cl}$ & Variada & $\begin{array}{l}\text { Masivo / } \\
\text { Alternativo }\end{array}$ \\
\hline
\end{tabular}

Fuente: Elaboración propia.

PP: Propiedad privada.

E: Ejido.

$\mathrm{Cl}$ : Comunidad indígena.

o segmentos del turismo con base principalmente en el diagnóstico y el peso específico de la actividad en la región, zona o municipio.

Cabe señalar que de los datos presentados en los cuadros 4 a II sólo se relacionaron aquellos recursos que se constataron directamente y que poseen cierta singularidad, el trabajo de campo de registro se realizó durante el periodo 1998-2003. 
Patrimonio, turismo y desarrollo sustentable: caso costa de Jalisco

Cuadro 3. Datos relacionados con el turismo de la región Costa Norte de Jalisco

\begin{tabular}{|c|c|c|c|c|}
\hline \multirow{2}{*}{ Zona } & PuertoVallarta & $\begin{array}{l}\text { Cabo Corrientes } \\
\text { y Tomatlán }\end{array}$ & $\begin{array}{l}\text { La Huerta } \\
\text { y Cihuatlán }\end{array}$ & \multirow[t]{2}{*}{$\begin{array}{l}\text { Región costa } \\
\text { de Jalisco }\end{array}$} \\
\hline & Costa norte & Costa centro norte & Costa sur & \\
\hline Hoteles & 154 & - & 69 & 223 \\
\hline Cuartos & 15098 & - & 2203 & 17301 \\
\hline $\begin{array}{l}\text { Turistas } \\
\text { hospedados }\end{array}$ & 1715630 & - & 284014 & I 999644 \\
\hline $\begin{array}{l}\text { Derrama (en } \\
\text { miles de pesos) }\end{array}$ & 261600589 & - & 13321760 & 274922349 \\
\hline Marinas & 1 & - & I & 2 \\
\hline $\begin{array}{l}\text { Agencias de } \\
\text { viaje }\end{array}$ & 67 & - & 16 & 83 \\
\hline
\end{tabular}

Fuente: INEGI (1998). Anuario estadístico del estado de Jalisco, México: Gobierno del Estado de Jalisco.

CUADRO 4. IDENTIFICACIÓN DE RECURSOS CULTURALES POR MUNICIPIO

\begin{tabular}{lrrrrrr}
\hline & $\begin{array}{c}\text { Puerto } \\
\text { Vallarta }\end{array}$ & $\begin{array}{c}\text { Cabo } \\
\text { Corrientes }\end{array}$ & Tomatlán & $\begin{array}{c}\text { La } \\
\text { Huerta }\end{array}$ & Cihuatlán & $\begin{array}{c}\text { Región } \\
\text { costa jal. }\end{array}$ \\
\hline Vestigios prehispánicos & 73 & 58 & 87 & 17 & 14 & 249 \\
Arquitectura colonial & 2 & 3 & 3 & 1 & 1 & 10 \\
Cultura viva & 14 & 25 & 8 & 12 & 11 & 70 \\
Arte & 9 & 1 & - & 1 & 2 & 13 \\
Eventos & 22 & 21 & 19 & 22 & 15 & 99 \\
programados & & 108 & 117 & 69 & 52 & 441 \\
Recursos culturales & 120 & 108 & & & & \\
\hline
\end{tabular}

Fuente: Elaboración propia. 
Una vez ponderados los recursos culturales bajo los criterios y valores establecidos, nos permiten su traslación o propuesta como atractivos turísticos, el resultado preliminar queda reflejado en el cuadro 5.

CUADRO 5. VALORACIÓN DE LOS RECURSOS IDENTIFICADOS COMO ATRACTIVO TURÍSTICO*

\begin{tabular}{|c|c|c|c|c|c|c|}
\hline $\begin{array}{l}\text { AsPECTOS } \\
\text { CUANTITATIVOS }\end{array}$ & $\begin{array}{l}\text { Puerto } \\
\text { Vallarta }\end{array}$ & $\begin{array}{c}\text { Cabo } \\
\text { Corrientes }\end{array}$ & Tomatlán & $\begin{array}{c}\text { La } \\
\text { Huerta }\end{array}$ & Cihuatlán & $\begin{array}{c}\text { Región } \\
\text { costa } \\
\text { Jal. }\end{array}$ \\
\hline $\begin{array}{l}\text { Vestigios } \\
\text { prehispánicos }\end{array}$ & 2 & 3 & $\mathrm{I}$ & $\mathrm{I}$ & - & 7 \\
\hline $\begin{array}{l}\text { Arquitectura } \\
\text { colonial }^{* *}\end{array}$ & - & I & 2 & I & I & 5 \\
\hline Cultura viva & 6 & 6 & I & 4 & 6 & 23 \\
\hline Arte & 1 & - & - & - & - & 1 \\
\hline Eventos programados & 5 & 1 & 2 & 1 & 2 & II \\
\hline Totales & 14 & II & 6 & 7 & 9 & 47 \\
\hline \multicolumn{7}{|l|}{$\begin{array}{l}\text { AsPECTOS } \\
\text { CUALITATIVOS *** }\end{array}$} \\
\hline Historicidad & 2 & 2 & 2 & I & 2 & 1.8 \\
\hline Paisaje cultural & 3 & 2 & I & 2 & 2 & 2.0 \\
\hline Espacios culturales & 3 & 1 & 1 & 2 & 2 & 1.8 \\
\hline Espacios testimoniales & 2 & 1 & 1 & 2 & 3 & 2.0 \\
\hline Totales & 2.5 & 1.7 & 1.2 & 1.7 & 2.2 & 1.9 \\
\hline
\end{tabular}

Fuente: Elaboración propia.

* Se presenta la resultante (recursos culturales cuantitativamente por variable) una vez que se ponderaron los elementos del cuadro 3 . Se utilizó la siguiente relación de valores $3=$ muy atractivo; 2 = atractivo; $I$ = poco atractivo.

**La única finca que hubiera sido posible recuperar y relacionarla con la actividad turística, la ex Hacienda del Pitillal, fue derribada a principios del 2004 con autorización del gobierno municipal y en su lugar se construyó un supermercado de la cadena Soriana.

***Dado que estas variables son estudiadas como unidad se puede ponderar directamente con los mismos valores asignados anteriormente ( 3 = muy atractivo; 2 = atractivo; $I$ = poco atractivo). 
CUADRO 6. IDENTIFICACIÓN DE RECURSOS NATURALES POR MUNICIPIO

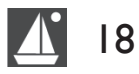

\begin{tabular}{|c|c|c|c|c|c|c|c|}
\hline Tipo & Recursos naturales & PV & $\subset$ & $\mathrm{T}$ & $\mathrm{H}$ & $\mathrm{a}$ & Región \\
\hline G & Playas & 11 & 22 & 10 & 12 & 8 & 63 \\
\hline G & Islas & 1 & $x$ & $x$ & 3 & $x$ & 4 \\
\hline G & Comunidades coralinas & 1 & 8 & $x$ & 3 & 4 & 16 \\
\hline G & Oquedades & $x$ & $x$ & $x$ & 5 & $x$ & 5 \\
\hline G & Montañas / cerros & 10 & 18 & 22 & II & 12 & 73 \\
\hline G & Bahías / caletas & $x$ & 6 & 2 & 4 & 5 & 17 \\
\hline G & Dunas & $x$ & $x$ & 1 & $x$ & $x$ & 1 \\
\hline H & Saltos / cascadas & 3 & 12 & 10 & 5 & $x$ & 30 \\
\hline $\mathrm{H}$ & Ríos /arroyos & 7 & 7 & 4 & 6 & 4 & 28 \\
\hline $\mathrm{H}$ & Presas & $x$ & $x$ & 1 & $x$ & $x$ & 1 \\
\hline $\mathrm{H}$ & Esteros /lagunas costeras & 2 & 5 & 8 & 9 & 3 & 27 \\
\hline H & Manantiales / aguas termales & 3 & 3 & 1 & 3 & $x$ & 10 \\
\hline B & Tipos vegetación & 3 & 7 & 5 & 6 & 5 & 26 \\
\hline B & Fauna (especies avifauna) & $*$ & 326 & $*$ & 98 & 62 & 486 \\
\hline \multirow[t]{2}{*}{ B } & Áreas Naturales Protegidas & 2 & $x$ & 1 & 5 & $x$ & 8 \\
\hline & Totales & 103 & & & & & 795 \\
\hline
\end{tabular}

Fuente: Elaboración propia. Patrimonio Natural de la Costa de Jalisco. Matriz de casos totales. Simbología:
$x=$ ausencia
$\mathrm{H}=$ La Huerta
$*$ = dato desconocido
$\mathrm{Cl}=$ Cihuatlán
$\mathrm{PV}=$ Puerto Vallarta
$\mathrm{G}=$ Tipo geológico
$\mathrm{cc}=$ Cabo Corrientes
$\mathrm{T}=$ Tomatlán
$\mathrm{H}=$ Hidrológico
$\mathrm{B}=$ Biológico

Las relaciones entre los recursos (naturales, culturales o productivos) se pueden establecer inter e intramunicipal, ya que se deriva a partir de la coincidencia temática o de la participación espacial, es decir, la concentración o dispersión de los mismos a partir del trazo de una posible ruta.

Lo anterior presupone, además, un trabajo paralelo en la atención de los posibles conflictos intermunicipales en el caso de darse la posibilidad de 
CUADRO 7. VALORACIÓN DE LOS RECURSOS IDENTIFICADOS COMO ATRACTIVO TURISTICO

\begin{tabular}{|c|c|c|c|c|c|c|c|}
\hline Tipo & $\begin{array}{l}\text { Recursos naturales con } \\
\text { potencial turístico }\end{array}$ & PV & $\mathrm{CC}$ & $\mathrm{T}$ & $\mathrm{H}$ & $\mathrm{Cl}$ & Región \\
\hline$g$ & Playas & 9 & 15 & 11 & 7 & 2 & 44 \\
\hline$g$ & Islas & $x$ & $x$ & 3 & $x$ & $x$ & 3 \\
\hline$g$ & Comunidades coralinas & $x$ & 5 & 3 & 3 & 1 & 12 \\
\hline$g$ & Oquedades & $x$ & $x$ & 2 & $x$ & $x$ & 2 \\
\hline$g$ & Montañas / cerros & 7 & 10 & 4 & 3 & 6 & 30 \\
\hline$g$ & Bahías / caletas & 2 & 6 & 4 & 5 & $x$ & 17 \\
\hline$g$ & Dunas & I & $x$ & $x$ & $x$ & $x$ & I \\
\hline $\mathrm{h}$ & Saltos / cascadas & 5 & 6 & 4 & $x$ & 4 & 19 \\
\hline $\mathrm{h}$ & Ríos / arroyos & 3 & 4 & 3 & 2 & 4 & 16 \\
\hline $\mathrm{h}$ & Presas & 1 & $x$ & $x$ & $x$ & $x$ & 1 \\
\hline $\mathrm{h}$ & Esteros / lagunas costeras & 5 & 4 & 5 & 3 & 2 & 19 \\
\hline $\mathrm{h}$ & Manantiales /aguas termales & $x$ & 2 & 3 & $x$ & 3 & 8 \\
\hline$b$ & Tipos vegetación & 5 & 7 & 6 & 5 & 3 & 26 \\
\hline$b$ & Fauna (especies avifauna) & $*$ & 256 & $*$ & 53 & 47 & 356 \\
\hline \multirow[t]{2}{*}{$b$} & Áreas Naturales Protegidas & 2 & $x$ & $\mathrm{I}$ & 5 & $x$ & 8 \\
\hline & Totales & 27 & 315 & 39 & 106 & 75 & 562 \\
\hline
\end{tabular}

Fuente: Elaboración propia. Patrimonio Natural de la Costa de Jalisco. Matriz de casos totales. Simbología:

$x=$ ausencia

$*$ = dato desconocido

$\mathrm{PV}=$ Puerto Vallarta

$\mathrm{cc}=$ Cabo Corrientes

$\mathrm{T}=$ Tomatlán

$$
\begin{aligned}
& \mathrm{H}=\text { La Huerta } \\
& \mathrm{Cl}=\text { Cihuatlán } \\
& \mathrm{G}=\text { Tipo geológico } \\
& \mathrm{H}=\text { Hidrológico } \\
& \mathrm{B}=\text { Biológico }
\end{aligned}
$$

un continuo espacial a través de un recurso ponderado y valorado como atractivo "regional".

Para el caso intramunicipal, estos posibles conflictos deberán ser resueltos a través de talleres participativos en los espacios públicos tradicionales: asamblea comunal, asamblea ejidal o participación ciudadana. 
CUADRO 8. IDENTIFICACIÓN DE RECURSOS PRODUCTIVO POR MUNICIPIO

\begin{tabular}{|c|c|c|c|c|c|c|c|}
\hline Actividad & Unidad & $\begin{array}{l}\text { Puerto } \\
\text { Vallarta }\end{array}$ & $\begin{array}{l}\text { Cabo } \\
\text { Corrientes }\end{array}$ & Tomatlán & $\begin{array}{l}\text { La } \\
\text { Huerta }\end{array}$ & Cihuatlán & Región \\
\hline \multirow{3}{*}{ Agricultura } & Ejido & 16 & 15 & 33 & 27 & 10 & 96 \\
\hline & $\begin{array}{r}\text { Comunidad } \\
\text { indígena }\end{array}$ & - & 5 & 2 & 2 & - & 9 \\
\hline & $\begin{array}{r}\text { Terreno } \\
\text { nacional }\end{array}$ & - & 2 & I & - & - & 3 \\
\hline \multirow{3}{*}{ Ganadería } & Ejido & 10 & 9 & 20 & 16 & 8 & 63 \\
\hline & $\begin{array}{r}\text { Comunidad } \\
\text { indígena }\end{array}$ & 2 & 5 & 2 & 2 & - & 11 \\
\hline & $\begin{array}{r}\text { Terreno } \\
\text { nacional }\end{array}$ & - & 2 & I & - & - & 3 \\
\hline \multirow[t]{2}{*}{ Pesca } & Ribereña & 4 & 6 & 6 & 9 & 8 & 33 \\
\hline & Continental & - & - & 1 & - & - & 1 \\
\hline \multirow{2}{*}{$\begin{array}{l}\text { Aprovecha- } \\
\text { miento } \\
\text { forestal }\end{array}$} & Silvicultura & - & I & - & - & - & I \\
\hline & Explotación & - & 1 & 1 & 1 & I & 4 \\
\hline \multirow[t]{2}{*}{ Minería } & Activa & I & - & - & - & - & 1 \\
\hline & Inactiva & - & - & - & 2 & - & 2 \\
\hline Totales & & 33 & 47 & 67 & 59 & 27 & 227 \\
\hline
\end{tabular}

Fuente: Elaboración propia.

CUADRO 9. VALORACIÓN DE LOS RECURSOS IDENTIFICADOS COMO ATRACTIVO TURÍSTICO

\begin{tabular}{lcccccc}
\hline Recurso Productivo & $\begin{array}{l}\text { Puerto } \\
\text { Vallarta }\end{array}$ & $\begin{array}{c}\text { Cabo } \\
\text { Corrientes }\end{array}$ & $\begin{array}{c}\text { Tomatlán } \\
\text { Huerta }\end{array}$ & $\begin{array}{c}\text { Cahuatlán } \\
\text { Región costa } \\
\text { Jalisco }\end{array}$ \\
\hline Agricultura & 6 & 5 & 12 & 10 & 8 & 41 \\
Ganadería & 0 & 1 & 10 & 7 & 0 & 18 \\
Pesca & 0 & 5 & 7 & 7 & 6 & 25 \\
$\begin{array}{l}\text { Aprovechamiento } \\
\text { forestal }\end{array}$ & 0 & 1 & 0 & 0 & 0 & 1 \\
Minería & 0 & 0 & 0 & 1 & 0 & 1 \\
Total & 6 & 12 & 29 & 25 & 14 & 86 \\
\hline
\end{tabular}

Fuente: Elaboración propia. 
CUADRO I0. POTENCIALIDAD TURISTICA POR MUNICIPIOS Y REGIÓN

\begin{tabular}{lcccccc}
\hline & \multicolumn{6}{c}{ Municipios } \\
\cline { 2 - 7 } Segmento turístico* & $\begin{array}{c}\text { Puerto } \\
\text { Vallarta }\end{array}$ & $\begin{array}{c}\text { Cabo } \\
\text { Corrientes }\end{array}$ & Tomatlán & $\begin{array}{c}\text { La } \\
\text { Huerta }\end{array}$ & $\begin{array}{c}\text { Región } \\
\text { Cihuatlán } \\
\text { Ecoturismo }\end{array}$ \\
Turismo de aventura & 1 & 3 & 3 & 3 & 2 & Jalisco \\
Turismo cultural & 3 & 3 & 2 & 3 & 3 & 2.4 \\
Etnoturismo & 3 & 1 & 2 & 1 & 2 & 1.8 \\
Turismo rural & 1 & 3 & 2 & 2 & 1 & 1.8 \\
Agroturismo & 1 & 3 & 2 & 2 & 2 & 2.0 \\
\hline
\end{tabular}

Fuente: Elaboración propia.

* Los valores asignados a estos rubros corresponden a la siguiente relación: 3 = alto; 2 = medio; I = bajo.

El cuadro 10 reúne los resultados preliminares de la inclusión de los elementos considerados en cada región según el tipo de recurso.

\section{Conclusiones}

El turismo como vínculo regional supone un estudio detallado de las fases del fenómeno previas al producto turístico como tal, por lo que se inició por la trípode que funge como base de dicha actividad: los recursos naturales, culturales y productivos. Por lo general, es común relacionar los conceptos de recurso con natural y cultural, sin embargo, las nuevas tendencias del turismo, más allá del simple y contrariado concepto de alternativo, presuponen una actividad cada vez más intelectual y de contacto con la realidad circundante; con el entorno natural y cultural, donde lo productivo es un híbrido o resultado de la incidencia de lo natural con lo cultural.

Por tanto, queda enmarcado en el estudio que los recursos naturales, culturales y productivos serán clasificados y ponderados desde su pertinencia turística, esto es, desde el ecoturismo, etnoturismo, turismo de aventura y el turismo rural (agroturismo para algunos autores; Mieckowski, 1995), en el caso del turismo masivo son otros factores a considerar, como la infraestructura $y$ la supraestructura turística (Boullón, 1995: 16; De la Torre, 1997: 58). 
Si bien se ha expuesto grosso modo el trabajo realizado, en esta etapa de la investigación podemos inferir la tipología turística con base en la existencia y valoración de los recursos, o en conjunto, del patrimonio municipal y de su expresión o traducción como viabilidad y potencialidad de uso e inserción como atractivos en la actividad turística, se concluye lo siguiente:

I. La región Costa Norte de Jalisco o Costa Alegre posee recursos tales que su implementación al mercado turístico requiere de un estudio particular donde se tomen en cuenta al menos tres variables: a) grado de interés y participación local; b) el modelo de incorporación de los bienes naturales, culturales y productivos a fin de establecer sus capacidades de carga, un programa de manejo y conservación; c) los procedimientos y estrategias de comercialización y operación; d) programa de capacitación especializada a guías locales en cuanto a segmentos turísticos alternativos; e) elaboración de políticas públicas en apoyo a proyectos específicos, determinación del grado de participación del sector privado (inversiones) y esquemas que garanticen la participación de la población local, más allá del simple concepto de empleado.

II. Que existen recursos tales que permiten un desarrollo que tienda a la sustentabilidad y que en la diversificación de los segmentos turísticos regidos por la ponderación de sus recursos, los municipios se desarrollen y contribuyan de manera significativa, estratégica y competitiva en el desarrollo regional, aprovechando el posicionamiento como destinos turísticos masivos de Puerto Vallarta y Barra de Navidad-Melaque y que el corredor a su vez complemente la oferta de dichos municipios para alargar la estancia turística partiendo de un concepto de sol y playa.

III. Fortalecer las identidades locales a partir de un apropiamiento y revaloración de sus recursos, que permita incluir actividades productivas a las ya existentes con el propósito de mejorar la calidad de vida y minimizar la huella ecológica en la región Costa Alegre de Jalisco.

\section{FUENTES CONSULTADAS}

Andrade-Romo, E. (2000). Caracterización del municipio de Cabo Corrientes, Jalisco, México. Suficiencia investigadora. México: Universidad de Guadalajara. 
Boullón, R. (1995). Proyectos turísticos: identificación, localización y dimensionamiento. México: Diana.

De la Torre Padilla, O. (1997). El turismo: fenómeno social. México: Fondo de Cultura Económica.

Everitt, J. et al. (200I)."Viva Vallarta! Impacts of the Re-definition of a Tourist Resort in Jalisco/Nayarit, Mexico". Prairie Perspectives, Geographical Essays, 4, 186-204.

Gobierno del Estado de Jalisco (2000a). Regionalización del estado de Jalisco, Región 8, Costa Sur, México: Gobierno del Estado de Jalisco.

-------- (2000b). Regionalización del estado de Jalisco, Región 9, Costa Sur, México: Gobierno del Estado de Jalisco.

INEGI/Gobierno de Jalisco (1998). Anuario estadístico del estado de Jalisco. Aguascalientes: Instituto Nacional de Estadística, Geografía e Informática.

López Guerrero, L. et al. (2002). “Un recorrido por la bahía de norte a sur”. México, 3, (I-2, 5-12).

Martínez Tarragó,T. (2000). Manual para la identificación, formulación y evaluación de empresas de turismo rural en México. México: Cestur.

Massam, B. H. et al. (2003). Quality of Life in the Puerto Vallarta Region of Jalisco State, Mexico. Canada: Rural Development Institute/Brandon University.

México (2002). "Ecología-Vallarta-Bahía de Banderas”, 3, (I-2). México: Universidad de Guadalajara.

Mieckowski, Z. (1995). Environmental issues of tourism and recreation, University Press of America, Lantarn, Maryland, Ecoturismo: Impactos, potencial y posibilidades, Wearing - Neil, 2000, Butterworth - Heinemann.

Sectur, (s/f). Termómetro turístico. México: Secretaría de Turismo.

UDG (1999). Proyecto caracterización de la Bahía de Banderas. México:

Universidad de Guadalajara.

UDG (2004). Vocacionalidad turística de la Costa de Jalisco. México:Universidad de Guadalajara. 


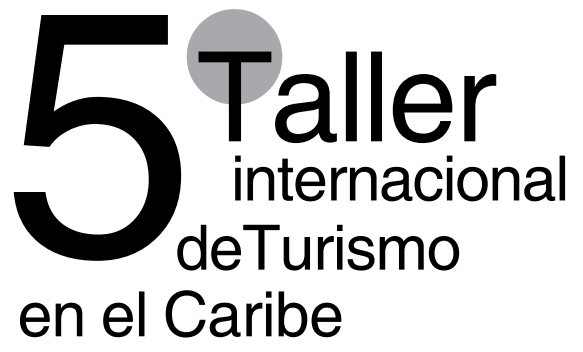

\section{La investigación} científica del turismo Isla de Cozumel, México 22 al 24 de noviembre 2006

La Universidad de Quintana Roo a través de la División de Desarrollo Sustentable, organizan el evento académico que pretende promover la discusión y reflexión interdisciplinaria en torno a las implicaciones sociales, culturales, económicas y ambientales del turismo, así como propuestas de orden teórico- metodológico para su investigación.

Conferencistas Invitados

Dra. Regina Schlüter, CIET/Argentina

Dr. Daniel Hiernaux-Nicolas, UAM-Iztapalapa

Informes:

Mtro. Alejandro Palafox Muñoz

Cuerpo Académico de Turismo Universidad de Quintana Roo vtaller@correo.uqroo.mx
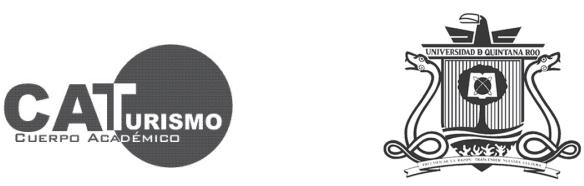